\title{
DESCRIPCIÓN DE DOS NUEVAS ESPECIES DE PSEUDOSCORPIONES CAVERNÍCOLAS DE LA PROVINCIA DE CÁDIZ (ARACHNIDA, PSEUDOSCORPIONIDA, CHTHONIIDAE, NEOBISIIDAE)
}

\author{
E. Carabajal Márquez (*), J. García Carrillo (**) \\ y F. Rodríguez Fernández (***)
}

\begin{abstract}
RESUMEN
Se describen dos nuevas especies de Pseudoscorpionida cavernícolas procedentes de la provincia de Cádiz (sur de España): Chthonius (Ephippiochthonius) aguileraorum sp. n. y Neobisium (Ommatoblothrus) rodrigoi sp. n., y se comparan con las especias más próximas.

Palabras clave: Arachnida, Pseudoscorpionida, Chthoniidae, Neobisiidae, Ephippiochthonius, Ommatoblothrus, cavernícola, especies nuevas, Villaluenga del Rosario, Cádiz, España.
\end{abstract}

\begin{abstract}
Description of two new species of cave-dwelling Pseudoscorpionida from Cádiz (South Spain) (Arachnida, Pseudoscorpionida, Chthoniidae, Neobisiidae)

Two new species of cave-dwelling Pseudoscorpionida from Cádiz (South Spain) are described: Chthonius (Ephippiochthonius) aguileraorum sp. n. and Neobisium (Ommatoblothrus) rodrigoi sp. $\mathrm{n}$., and it is compared with the close relatives species.

Key words: Arachnida, Pseudoscorpionida, Chthoniidae, Neobisiidae, Ephippiochthonius, Ommatoblothrus, cave dwelling, new species, Villaluenga del Rosario, Cádiz, Spain.
\end{abstract}

\section{Introducción}

Durante una corta excursión espeleológica en la Sierra de Grazalema (Cádiz), en la que nuestro objetivo primario era el de recolectar algún ejemplar de Neobisium (Ommatoblothrus) gaditanum Mahnert, 1977, con el fin de obtener material de comparación, visitamos dos cavidades en el término municipal de Villaluenga del Rosario: la Cueva del Cacao en la que pudimos capturar una tritoninfa de la mencionada especie, y la Cueva de la
Hiedra, situada a poca distancia de la anterior y en la que se capturaron varios ejemplares de dos nuevas especies de Pseudoscorpionida, que a continuación se describen.

\section{Material y métodos}

Los ejemplares estudiados fueron localizados visualmente y capturados mediante el uso de un aspirador, posteriormente conservados en alcohol

\footnotetext{
* Alcalde Garrido Juaristi 15-5ㄷ. 28030 - Madrid (ESPAÑA); carabajal@mad.servicom.es

** $\quad$ Portalegre 104- $2^{\circ} \mathrm{B}, 28019$ - Madrid (ESPAÑA); jgarcia@microdelta.es

*** San Andrés 10-2 G, 28220 - Majadahonda, Madrid (ESPAÑA); F.Rodriguez.Fernandez@mad.servicom.es
} 
etílico de $70^{\circ}$, las medidas fueron tomadas mediante micrómetro ocular y los dibujos fueron realizados mediante cámara clara acoplada a un microscopio óptico, la nomenclatura es la estándar utilizada por Beier (1963). El símbolo "X" se utiliza como equivalente a "veces más que...".

\section{Chthonius (Ephippiochthonius) aguileraorum} sp. n. (figs. 1-4) (tabla 1)

SERIE TíPICA. Holotipo, 우 . Cueva de la Hiedra, Villaluenga del Rosario, Cádiz, España, 04-VII-1998, E. Carabajal \& J Aguilera leg. (en las colecciones del Museo Nacional de Ciencias Naturales de Madrid, $\mathrm{n}^{\circ}$ de catálogo: $\mathrm{MNCN}$ 20.02/9960).

Paratipos: 2 우 ㅇ, misma localidad, fecha y recolectores, 1 tritoninfa y 2 deutoninfas, misma localidad, 08-XI-1998 y 3 우 우, 2 tritoninfas y 2 deutoninfas, misma localidad 01-IV1999 E. Carabajal, J. García \& F. Rodríguez leg. en las colecciones E. Carabajal, J. García y V. Mahnert del Museo de Historia Natural de Ginebra.

DiAGNOSIS. Gran tamaño, facies cavernícola, despigmentado y de apéndices alargados (mano del pedipaldo 2,1 veces más larga que ancha, quela 5,2 veces). Cefalotórax con 20 sedas, 4 de ellas en el borde posterior, con ojos delanteros separados de las manchas oculares traseras por la distancia de 2 diámetros oculares. Quelíceros con un diente subapical aislado en el dedo móvil. Dedo fijo de la pinza del pedipalpo con 24-26 dientes.

DESCRIPCIÓN: Longitud: $2,75 \mathrm{~mm}$ sin contar los quelíceros; $3,35 \mathrm{~mm}$ longitud total

Cefalotórax: de igual longitud que anchura $(0,70 \times 0,70 \mathrm{~mm})$, estrechado en el borde anterior $(0,65 \mathrm{~mm})$, con su máxima anchura $(0,70 \mathrm{~mm})$ a nivel de la zona ocular y posteriormente estrechándose de modo progresivo hacia el borde posterior $(0,60 \mathrm{~mm})$; borde anterior casi recto, en leve curva, y dentado en su zona media; con dos ojos a cada lado, muy reducidos, los delanteros con pequeña lente, los traseros reducidos a una mancha, refringente vista a $45^{\circ}$ desde atrás, y separados de los delanteros por la distancia de dos diámetros oculares; con 20 sedas $(4 / 6 / 4 / 2 / 4)+2$ microsedas preoculares a cada lado, de las 4 sedas posteriores las dos externas más pequeñas que las internas.

Quetotaxia de los terguitos I-IX: 4/4/4/4/6/6/6/6/6.

Quelícero (fig. 2): con 6 sedas +1 microseda en la mano; dedo fijo con 10 dientes: distalmente 1 de gran tamaño, posteriormente $1 / 2$ menores, luego 2 de gran tamaño y posteriormente 5 más pequeños $(1+1 / 2+2+5)$; dedo móvil con 1 diente distal de

Tabla 1.-Dimensiones (en mm) de la serie típica de Chthonius (Ephippiochthonius) agileraorum sp. n.

Table 1.- Measurements (in $\mathrm{mm}$ ) of the type material of Chthonius (Ephippiochthonius) agileraorum sp. $\mathrm{n}$.

\begin{tabular}{|c|c|c|c|c|c|}
\hline \multirow{2}{*}{\multicolumn{2}{|c|}{ Longitud del cuerpo (sin quelíceros) }} & Holotipo & Paratipos & Deutoninfa & Tritoninfa \\
\hline & & 2,75 & 2,26 a 2,80 & 1,24 & 1,70 \\
\hline \multicolumn{2}{|c|}{ Longitud total } & 3,35 & 2,73 a 3,40 & 1,54 & 1,99 \\
\hline \multicolumn{2}{|l|}{ Cefalotórax } & $0,70 / 0,70$ & $0,65 / 0,65$ a $0,70 / 0,64$ & $0,38 / 0,38$ & $0,45 / 0,45$ \\
\hline Pedipalpos & $\begin{array}{l}\text { Quela } \\
\text { Fémur } \\
\text { Tibia } \\
\text { Mano } \\
\text { Dedo }\end{array}$ & $\begin{array}{c}1,87 \\
1,35 / 0,15 \\
0,47 / 0,20 \\
0,75 / 0,28 \\
1,12\end{array}$ & $\begin{array}{c}1,68 \text { a } 1,84 \\
1,24 / 0,15 \text { a } 1,29 / 0,14 \\
0,45 / 0,19 \text { a } 0,48 / 0,20 \\
0,67 / 0,29 \text { a } 0,72 / 0,29 \\
1,03 \text { a } 1,12\end{array}$ & $\begin{array}{c}0,76 / 0,13 \\
0,54 / 0,08 \\
0,21 / 0,10 \\
0,32 / 0,13 \\
0,45\end{array}$ & $\begin{array}{c}1,04 / 0,18 \\
0,76 / 0,11 \\
0,28 / 0,14 \\
0,39 / 0,18 \\
0,65\end{array}$ \\
\hline \multicolumn{2}{|c|}{$\mathrm{N}^{\circ}$ dientes dedo fijo/móvil } & 25/9+1 tubérculo & 26/11+1 tubérculo & $17 / 7$ & 24/8+3 tubérculos \\
\hline Pata I & $\begin{array}{l}\text { mur-I } \\
\text { mur-II } \\
\text { ia } \\
\text { so }\end{array}$ & $\begin{array}{c}0,70 / 0,10 \\
0,29 \\
0,44 \\
0,77\end{array}$ & $\begin{array}{c}0,65 / 0,09 \text { a } 0,69 / 0,09 \\
0,31 / 0,09 \text { a } 0,35 / 0,08 \\
0,39 \text { a } 0,39 \\
0,73 \text { a } 0,74\end{array}$ & $\begin{array}{l}0,28 \\
0,12 \\
0,15 \\
0,32\end{array}$ & $\begin{array}{l}0,44 \\
0,17 \\
0,21 \\
0,39\end{array}$ \\
\hline Pata IV & $\begin{array}{l}\text { mur } \\
\text { ia } \\
\text { so-I } \\
\text { so-II }\end{array}$ & $\begin{array}{c}1,10 / 0,35 \\
0,67 / 0,11 \\
0,35 \\
0,79\end{array}$ & $\begin{array}{c}0,99 / 0,37 \text { a } 1,08 / 0,33 \\
0,65 / 0,11 \text { a } 0,67 / 0,13 \\
0,35 \text { a } 0,35 \\
0,71 \text { a } 0,79\end{array}$ & $\begin{array}{c}0,41 / 0,15 \\
0,26 / 0,06 \\
0,15 \\
0,29\end{array}$ & $\begin{array}{c}0,57 / 0,21 \\
0,37 / 0,09 \\
0,23 \\
0,44\end{array}$ \\
\hline
\end{tabular}


1

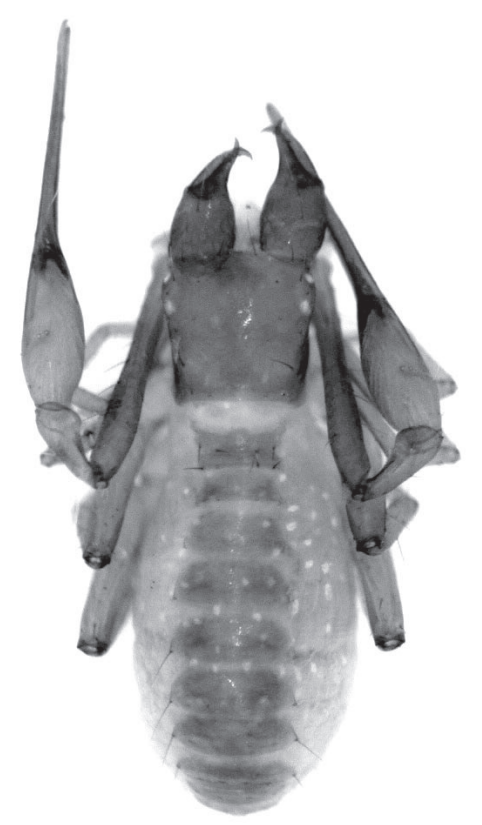

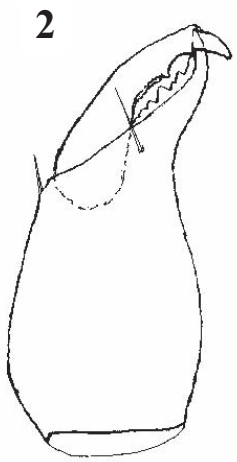

4

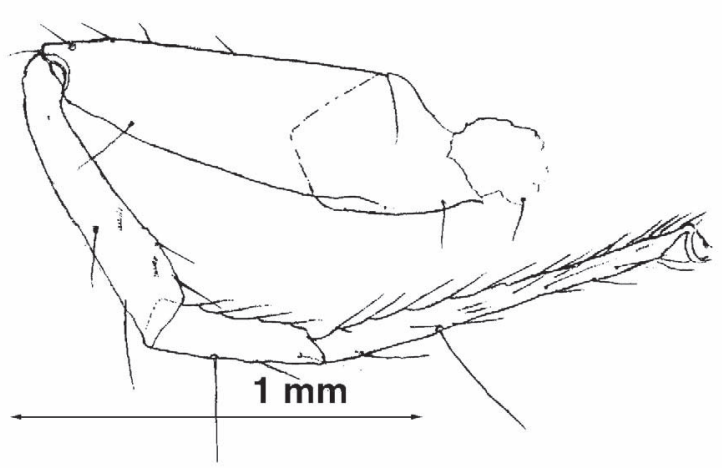

3a
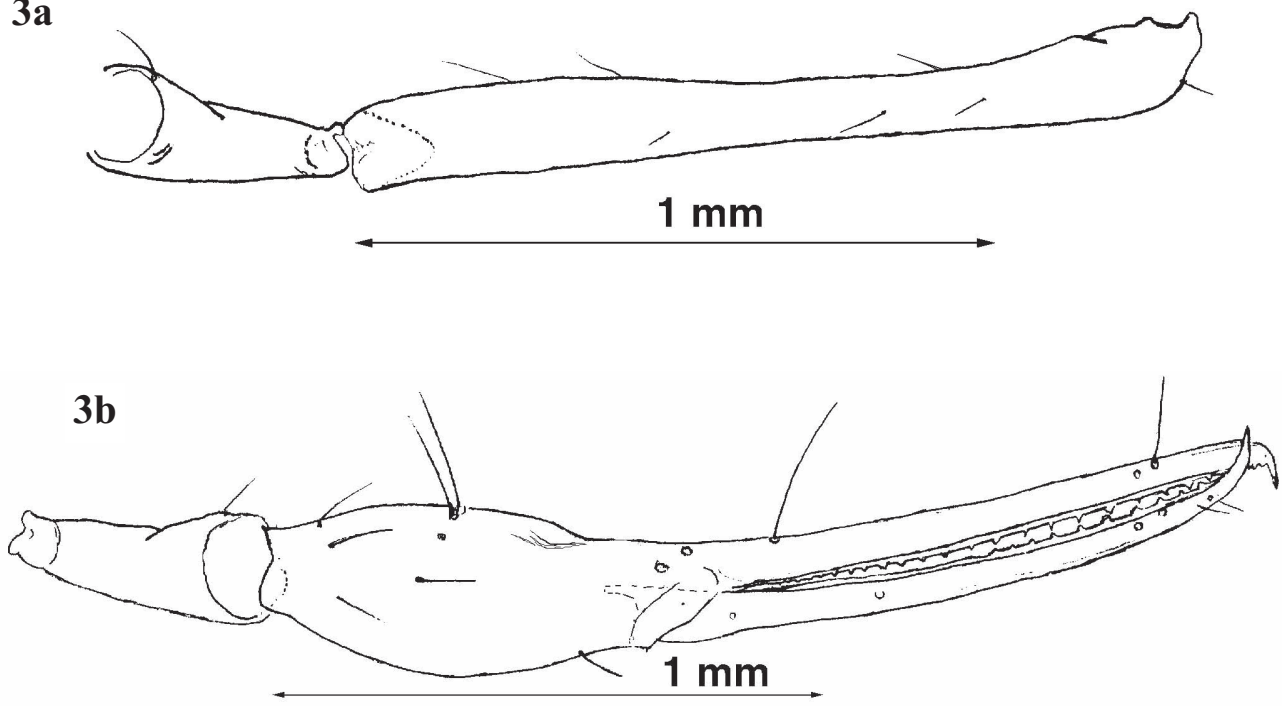

Figs. 1-4.-Chthonius (Ephippiochthonius) aguileraorum sp.n. 1) Holotipo; 2) Quelícero; 3a) Fémur y tibia del pedipalpo; 3b) Quela; 4) Pata IV.

Figs. 1-4.- Chthonius (Ephippiochthonius) aguileraorum sp.n. 1) Holotype; 2) Chelicerae; 3a) Femur and tibia of pedipalp; 3b) Chelae; 4) Leg IV. 
gran tamaño, y 5-7 más pequeños $(1+7)$ presenta además un diente subapical aislado; tubérculo sericígeno en forma de tubérculo romo; sérrula externa con 16 láminas, interna con 13; flagelo con 9 sedas pectinadas.

Lóbulo de la coxa del pedipalpo con 2 sedas, coxa del pedipalpo con 3 , coxa I con $3+3$ microsedas apicales y marginales, coxa II con 4 sedas +19 espinas coxales aserradas en sus $2 / 3$ apicales, coxa III con 5 sedas $+3-9$ espinas coxales aserradas en sus $2 / 3$ apicales, coxa IV con 7 , tubérculo intercoxal con 2 sedas.

Opérculo genital en la hembra con 10-11 sedas. Quetotaxia de los esternitos III-X: esternito III $10+6(2 \times 3)$ microsedas estigmales, IV: $8+2(2 \times 1)$ microsedas estigmales, $\mathrm{V}: 6+2$ sedas laterales diminutas, VI y VII: $4+2$ sedas laterales diminutas, VII y IX: $6, X: 7$ con 2 submedianas táctiles.

Pedipalpos (figs. 3a y $3 \mathrm{~b}$ ): trocánter 1,82 X más largo que ancho en la hembra; fémur $9 \mathrm{X}$ más largo que ancho, 2,87 X más largo que la tibia y 1,92 X más largo que el cefalotórax; tibia 2,35 X; mano 2,67 X, dedo móvil 1,49 X más largo que la mano; dedo fijo con 25 dientes, los distales y los cercanos a la base menos separados, los medios muy separados, dedo móvil con 9+1 distal, con lámina basal; tricobotrio ist muy separado de esb, et a la altura del 3er diente basal, $t$ basal del $5^{\circ}$ diente distal, $s t$ basal del $6^{\circ}$ distal, $b$ basal de ist y distal de $e s b$, distancia entre $s t$ y $s b 2 \mathrm{X}$ de la distancia entre $s b$ y $b$.

Patas marchadoras: Pata I: fémur I $7 \mathrm{X}$ más largo que ancho y 2,41 X más largo que el femur II; pata IV (fig. 4): 3,14 X; seda táctil (ST) del tarso I basal 0,36, ST del tarso II basal 0,31.

Etimología: Dedicamos esta nueva especie a los hermanos José y Julio Aguilera del GIEX de Jerez de La Frontera, que tan amablemente prestaron su colaboración y aportaron sus conocimientos sobre las cavidades de la zona y gracias a los cuales ha sido posible el descubrimiento de esta nueva especie.

Discusión: Chthonius (E.) aguileraorum sp. n. se diferencia a primer golpe de vista del resto de los Ephippiochthonius Beier, 1930 ibéricos que poseen ojos más o menos funcionales, por sus apéndices muy largos en proporción, así como por su gran tamaño (hasta 3,40 mm de longitud total), se separa de Chthonius (E.) hispanus Beier, 1930, única especie oculada presente en la Península Ibérica con la que, por su tamaño, podría confundirse, tanto por las diferentes proporciones de la mano del pedipalpo $(2,1 \mathrm{X})$ y quela $(5,2 \mathrm{X})$ como por el perfil de la misma y la posición de los tricobotrios, especialmente ist, así como por la ausencia en hispanus de diente subapical aislado en el dedo móvil del quelícero, difiere tambien en la quetotaxia del cefalotórax, 20 sedas en aguileraorum sp. n. y 18 en hispanus.

En cuanto a especies con 20 setas en el cefalotórax, únicamente Chthonius (E.) catalonicus Beier, 1939 y Chthonius (E.) cabreriensis Mahnert, 1993 comparten este carácter, ambas de mucho menor tamaño y con una dentición muy diferente, así como muchos otros e importantes detalles que no consideramos necesario relacionar.

\section{Neobisium (Ommatoblothrus) rodrigoi sp. $\mathrm{n}$.} (figs. 5-8) (tabla 2)

SERIE tíPICA. Holotipo, ơ . Cueva de la Hiedra, Villaluenga del Rosario, Cádiz, España, 04-VII-1998, E. Carabajal \& J. Aguilera leg. (en la Coll. E. Carabajal).

Paratipos: $1 \sigma^{x}$ y 1 \% , misma localidad y recolectores, depositados en la Coll. J. García y en las colecciones del Museo Nacional de Ciencias Naturales de Madrid, $n^{\circ}$ de catálogo: MNCN 20.02/9961, y 1 tritoninfa, misma localidad 08XI-1998 E. Carabajal, J. García \& F. Rodríguez leg. en la Coll. E. Carabajal.

DiAgNosis: Gran tamaño, con facies cavernícola, con los apéndices alargados (fémur del pedipalpo 5,7 veces más largo que ancho) y parcialmente despigmentado. Cefalotórax provisto de un epistoma triangular y agudo, con 22 sedas, 6 de ellas en la base. Dentición del dedo móvil de la quela uniforme, alcanzando la porción basal del mismo. Proceso antero-medial de la coxa I prominente y alargado. Quelícero con 6 sedas.

DESCRIPCIÓN: Longitud total: 4,50 $\mathrm{mm}$ macho, 5,20 mm hembra; sin contar los quelíceros: 4,00$4,60 \mathrm{~mm}$.

Cefalotórax: ligeramente más largo que ancho en el macho $(0,95 \times 0,85 \mathrm{~mm})$ con el borde anterior algo más estrecho $(0,70 \mathrm{~mm})$, la máxima anchura a nivel medio $(0,85 \mathrm{~mm})$ y apenas estrechado en su borde posterior $(0,82 \mathrm{~mm})$, provisto de un epistoma triangular y agudo; dos pares de ojos a cada lado, los delanteros provistos de lente, aunque blanquecinos y los traseros reducidos a una mancha y de menor diámetro; con 22 sedas (4/6/6/6) en ambos sexos, no presenta sedas preoculares.

Quetotaxia de los terguitos I-IX: 6-4/6-7/7-9/1011/11-12/12-12/11-12/12-13/10-11.

Quelícero (figs. 6a y 6b): con 7 sedas en la mano y 1 subgaleal en el tercio distal del dedo móvil; 

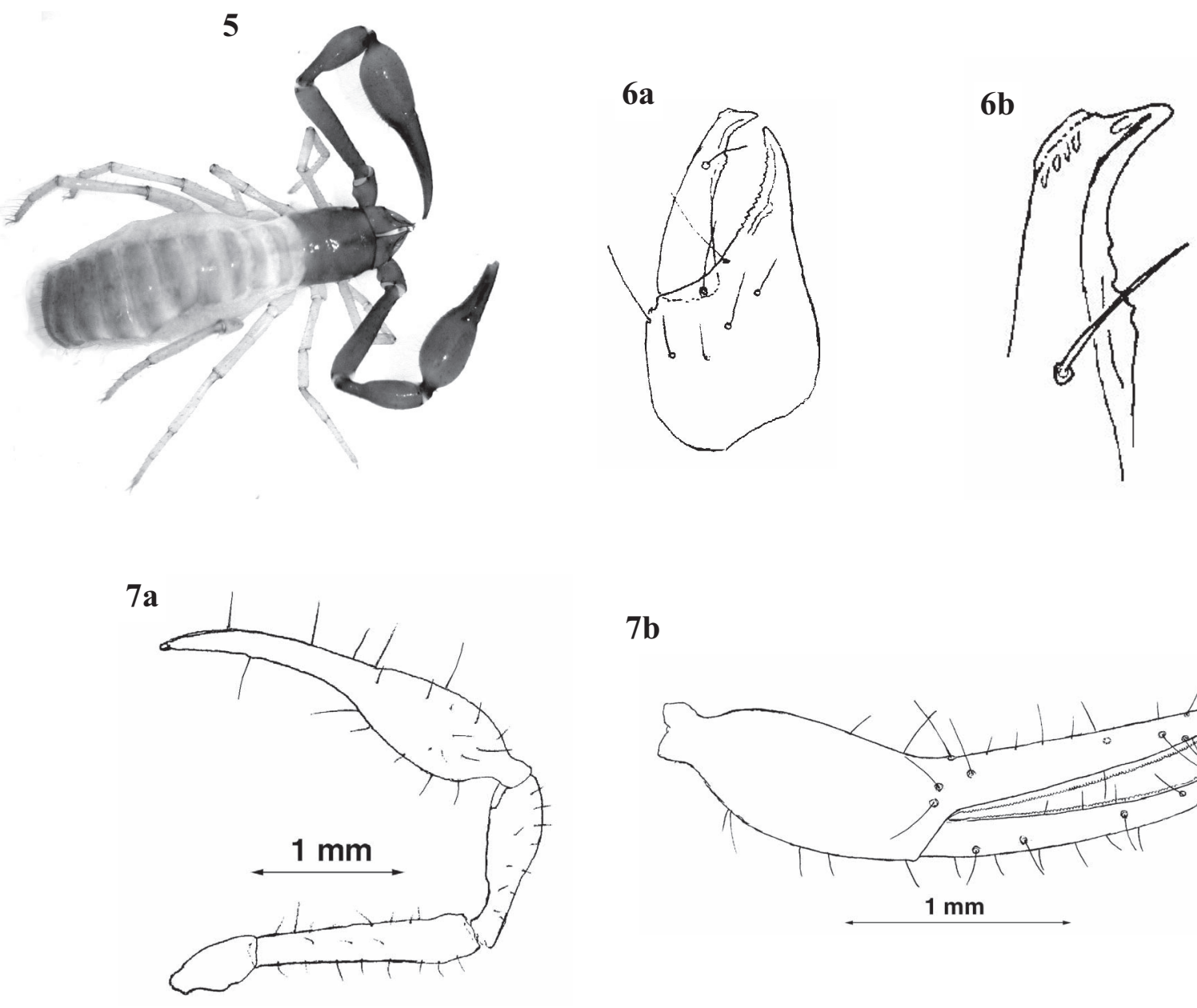

$7 \mathbf{b}$

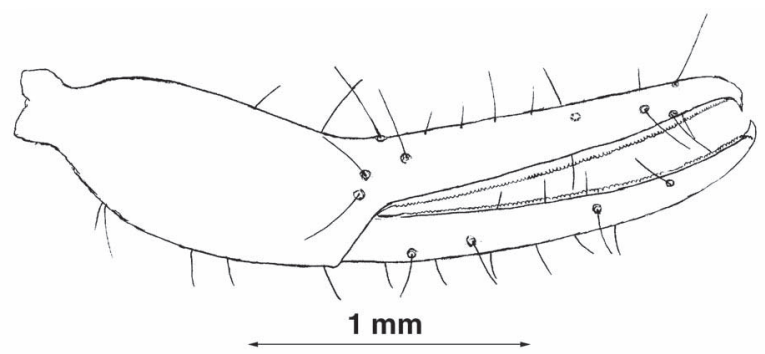

8

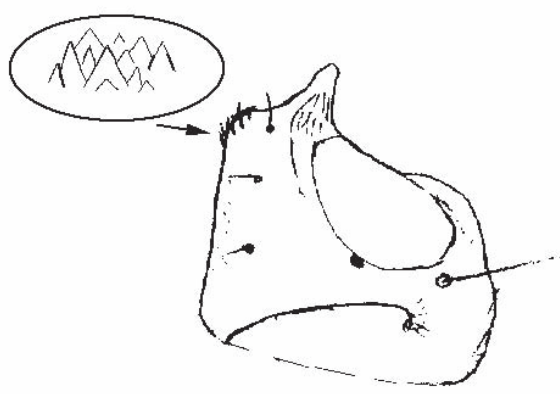

Figs. 5-8.- Neobisium (Ommatoblothrus) rodrigoi sp.n.: 5) Paratipo hembra; 6a) Quelícero; 6b) Detalle del dedo móvil del quelícero; 7a) Pedipalpo derecho; 7b) Quela; 8) Coxa de la pata I.

Figs. 5-8.- Neobisium (Ommatoblothrus) rodrigoi sp.n.: 5) Female paratype; 6a) Chelicerae; 6b) Detail of movable finger of the chelicerae; 7a) Right pedipalp; 7b) Chelae; 8) Coxae of leg I. 
Tabla 2.- Dimensiones (en $\mathrm{mm}$ ) de parte de la serie típica de Neobisium (Ommatoblothrus) rodrigoi sp. $\mathrm{n}$.

Table 2.- Measurements (in $\mathrm{mm}$ ) of part of the type material of Neobisium (Ommatoblothrus) rodrigoi sp. $\mathrm{n}$.

\begin{tabular}{|c|c|c|c|c|}
\hline \multirow{2}{*}{\multicolumn{2}{|c|}{ Longitud (sin contar quelíceros) }} & Holotipo & Paratipo 우 & Tritoninfa \\
\hline & & 4,00 & 4,60 & 2,70 \\
\hline \multicolumn{2}{|c|}{ Longitud total } & 4,40 & 5,15 & 3,20 \\
\hline \multicolumn{2}{|c|}{ Cefalotórax } & $0,95 / 0,85$ & $1,00 / 0,99$ & $0,78 / 0,73$ \\
\hline Pedipalpo & $\begin{array}{l}\text { Quela (con pedúnculo) } \\
\text { Fémur } \\
\text { Tibia } \\
\text { Mano } \\
\text { Dedo móvil } \\
\text { Dedo fijo }\end{array}$ & $\begin{array}{c}2,55 \\
1,55 / 0,27 \\
1,15 / 0,35 \\
1,15 / 0,55 \\
1,47 \\
1,40\end{array}$ & $\begin{array}{c}2,59 \\
1,50 / 0,30 \\
1,19 / 0,38 \\
1,15 / 0,60 \\
1,45 \\
1,40\end{array}$ & $\begin{array}{c}1,81 \\
1,05 / 0,22 \\
0,79 / 0,23 \\
0,71 / 0,45 \\
1,03 \\
1,03\end{array}$ \\
\hline Pata I & $\begin{array}{l}\text { mur I } \\
\text { mur II } \\
\text { ia } \\
\text { so I } \\
\text { so II }\end{array}$ & $\begin{array}{l}0,82 / 0,14 \\
0,52 / 0,14 \\
0,58 / 0,11 \\
0,44 / 0,10 \\
0,50 / 0,09\end{array}$ & $\begin{array}{l}0,82 / 0,14 \\
0,54 / 0,14 \\
0,62 / 0,12 \\
0,38 / 0,09 \\
0,52 / 0,09\end{array}$ & $\begin{array}{l}0,57 / 0,11 \\
0,34 / 0,11 \\
0,36 / 0,09 \\
0,24 / 0,08 \\
0,34 / 0,49\end{array}$ \\
\hline Pata IV & $\begin{array}{l}\text { mur I } \\
\text { mur II } \\
\text { ia } \\
\text { so I } \\
\text { so II }\end{array}$ & $\begin{array}{l}0,61 / 0,29 \\
0,73 / 0,29 \\
1,22 / 0,15 \\
0,57 / 0,11 \\
0,67 / 0,10\end{array}$ & $\begin{array}{l}0,65 / 0,30 \\
0,73 / 0,30 \\
1,17 / 0,14 \\
0,57 / 0,11 \\
0,64 / 0,11\end{array}$ & $\begin{array}{r}0,45 / 0,23 \\
0,47 / 0,23 \\
0,78 / 0,15 \\
0,37 / 0,09 \\
0,49 / 0,089\end{array}$ \\
\hline
\end{tabular}

dedo fijo con 18 dientes, de los cuales los 3-5 centrales mucho mayores; dedo móvil con una lámina ensanchada en su parte media, formada en su parte proximal por un gran diente aserrado con 1 a 3 vértices y distalmente con 5 pequeños dientes más, con tubérculo sericígeno en forma de media luna en ambos sexos; sérrula externa con 31 láminas; interna con 21 láminas; flagelo característico del género con 8 sedas, las 2 distales pectinadas en un lado y la última situada en una pequeña elevación y ligeramente separada de las otras.

Lóbulo de la coxa del pedipalpo con 4 sedas en el macho, 5 en la hembra, coxa del pedipalpo con 6-6 sedas, coxa I con 5-6, coxa II con 6-6, coxa III con 7-7, coxa IV con 8-11.

Región genital: Opérculo genital anterior con 812 sedas (macho)/8 (hembra), cámara con $2 \mathrm{X} 9$ sedas, opérculo posterior con 43 sedas (macho)/28 (hembra).

Quetotaxia de los esternitos III-X: 14-18/14-15 /16-18 /16-16 /16-16 /15-15/16-14/anal-anal.

Pedipalpos (figs, 7 a y $7 \mathrm{~b}$ ): trocánter $2 \mathrm{X}$ más largo que ancho en el macho; fémur 5,16 X, 1,36 X más largo que la tibia y 1,63 X más largo que el cefalotórax; tibia 3,28 X; mano 1,90 X; dedo móvil $1,4 \mathrm{X}$ más largo que la mano; quela $4,97 \mathrm{X}$.
Patas marchadoras: pata IV: seda táctil (ST) 0,39 en el macho y 0,37 en la hembra.

ETIMología: Dedicamos esta nueva especie a Rodrigo Carabajal, hijo de uno de los autores, que con tanta ilusión nos acompaña en nuestros muestreos en cavidades.

Discusión: Del subgénero Ommatoblothrus Beier, 1956 se han citado en el ámbito iberobalear únicamente dos especies hasta la fecha: Neobisium (O.) paucedentatum (Mahnert, 1982) de la Cueva del Agua, Iznalloz, Granada y Neobisium (O.) gaditanum (Mahnert, 1977) de la Sima del Cacao, Villaluenga del Rosario, Cádiz (Beier, 1963), (Mahnert, 1977), (Mahnert, 1982). Del primero de ellos, se diferencia por la quetotaxia del cefalotórax, que en paucedentatum presenta 10-16 sedas en la base, mientras que en rodrigoi sp. n., únicamente presenta 6 , diferente también es la dentición del dedo móvil de la quela que en rodrigoi se presenta completa hasta la porción basal del dedo, mientras que en paucedentatum únicamente es neta en los dos tercios distales; en cuanto a gaditanum, su aspecto, mucho mas evolucionado, es ya muy diferente, se trata de una especie de menor tamaño $(2,5-$ 
$3 \mathrm{~mm}$ ), con 8 setas en el borde posterior del cefalotórax, el tricobotrio ist se sitúa a la altura de $t$, mientras que en rodrigoi $\mathrm{sp}$. $\mathrm{n}$. lo hace a nivel de st.

Por otra parte, dentro del grupo de Ommatoblothrus que poseen 6 sedas en el borde basal del cefalotórax, se diferencia de Neobisium (O.) sardoum (Beier, 1956) de la Isla de Cerdeña por la diferente dentición del dedo fijo del pedipalpo, que en sardoum alterna un diente largo y uno corto, así como en el fémur mucho mas largo. De Neobisium (O.) cerruttii (Beier, 1955) de Lazio (Italia), por la diferente quetotaxia de la mano del quelícero, 7 sedas frente a 6 en rodrigoi sp. n., y por tener este último el fémur más corto que el dedo del pedipalpo. De Neobisium (O.) battonii (Beier, 1966) de Abruzzi (Italia) por el proceso anteromedial de la coxa I, que es redondeado en battonii, prominente y alargado en rodrigoi sp. n. (fig. 8). Difiere además de todos ellos a un tiempo por las diferentes proporciones de la quela, poco estilizada en la nueva especie (Gardini \& Rizzerio, 1986).

CONSIDERACIONES ECOLÓGICAS: Las dos especies aquí descritas fueron capturadas a escasos metros de la boca de entrada, en zona de penumbra y en las zonas de pared más blancas, destacando el hecho de la captura en asociación parietal de Chthonius (Ephippiochthonius) aguileraorum sp. n., ya que la gran mayoría de especies del subgénero se capturan bajo piedras, aún en medio subterráneo.

\section{Referencias}

BeIER, M., 1963. Ordnung Pseudoskorpionidea (Afterskorpione). Akademie Verlag, Bestimmungsbucher zur Bodenfauna Europas, 1. Berlin. 313 pp.

GardinI, G. \& Rizzerio, R., 1986. Neobisium (O.) zoiai n. sp. delle alpi liguri e note su Roncus ligusticus Beier, 1930. Bollettino della Società Entomologica Italiana, Genova, 118(1-3): 5-16.

MAHNERT, V., 1977. Spanische Hölenpseudoskorpione. Miscelánia Zoológica, 4(1): 61-104.

MAHNERT, V., 1982. Neue höhlenbewohnende Pseudoskorpione aus Spanien, Malta und Griechland (Arachnida, Pseudoscorpiones). Bulletin de la Société Entomologique Suisse, 55: 297-304.
Recibido, el 20-IX-1999

Aceptado, el 27-IX-2000 Publicado, el 27-XII-2000 